\title{
Difficult Intubation Provokes Bacteremia
}

\author{
Evangelos Konstantinou, ${ }^{1,2}$ Eriphili Argyra, ${ }^{2}$ Alexandra Avraamidou, ${ }^{3}$ Theofanis Fotis, ${ }^{1}$ \\ Maria Tsakiri, ${ }^{4}$ Dionisios Voros, ${ }^{5}$ and George Baltopoulos ${ }^{6}$
}

\begin{abstract}
Purpose: To evaluate the prevalence of bacteremia after mask ventilation, laryngoscopy, and endotracheal intubation before induction of general anesthesia and to discover any correlation between traumatic manipulations and bacteremia. The specific bacteria responsible, knowledge of which may guide the prophylactic use of antibiotics, also were investigated.

Methods: Fifty patients were enrolled. Three 10-mL blood samples were collected from a peripheral vein 10 min before induction of anesthesia, $10 \mathrm{~min}$ after mask ventilation, and $10 \mathrm{~min}$ after intubation. All samples were placed in aerobic and anaerobic bottles for culture and bacterial identification.

Results: Cultures received $10 \mathrm{~min}$ after intubation were positive in $12 \%$ of patients. The following strains were isolated: Escherichia coli in two cases, Staphylococcus aureus in three cases, and Peptostreptococcus anaerobius in one case. A strong positive correlation was found between difficult intubation and bacteremia. No correlation between bacteremia and easy intubation or between bacteremia and face mask ventilation was identified.

Conclusion: Traumatic manipulations during difficult laryngoscopy and endotracheal intubation could cause bacteremia. This finding may justify and guide prophylactic use of antibiotics.
\end{abstract}

\section{Introduction}

Colonization of the tracheobronchial tree happens often during or after orotracheal or nasotracheal intubation, tracheostomy, or long-term ventilatory care. Disruption of the natural bacterial barrier of the oropharynx or trachea is a risk factor for bacteremia [1]. Orotracheal intubation was implicated as a cause of bacteremia in $0-5.3 \%$ of adult patients in an earlier study [2]. In the pediatric population, major procedures such as rigid tracheobronchoscopy appear to be low risk [3]. In recent studies involving other interventions through the oral cavity such as transesophageal echocardiography, the incidence of bacteremia was significant [4]. Although routine antibiotic prophylaxis is not advocated prior to or during these procedures, it should be used in high-risk patients such as those with prosthetic valves, multivalvular disease involvement, or a history of infective endocarditis $[3,5]$.

In previous studies, the use of aseptic techniques and equipment during induction of anesthesia and intubation was not described clearly. We studied the possible occurrence and incidence of bacteremia after orotracheal intubation using single-use and sterilized equipment for ventilatory support. We also investigated the relation between difficult or traumatic manipulations of the tracheobronchial tree and bacteremia.

\section{Patients and Methods}

The study was conducted in the operating room of the Evgenidion General Hospital, National and Kapodistrian University of Athens, between October 1 and December 1, 2006. The protocol was approved by the hospital's Ethics Committee. Fifty patients aged 20-80 years, American Society of Anesthesiologists class I or II, who underwent elective surgery were enrolled. Patients with a history of diabetes, coronary artery or valvular heart disease, cerebrovascular disease, respiratory autoimmune disease, renal failure, or hemophilia were excluded. We also excluded immunocompromised patients, patients with myasthenia gravis, those

\footnotetext{
${ }^{1}$ Faculty of Nursing, National and Kapodistrian University of Athens, Athens, Greece.

${ }^{2}$ Department of Anesthesiology, National and Kapodistrian University of Athens, Athens, Greece.

${ }^{3}$ Department of Anaesthesia, Tzanio Hospital, Athens, Greece.

${ }^{4}$ Department of Microbiology, Evgenidion Hospital Athens, Athens, Greece.

${ }_{5}^{5}$ Department of Surgery, Medical School, National and Kapodistrian University of Athens, Athens, Greece.

${ }^{6}$ Department of Critical Care and Pulmonary Diseases, National and Kapodistrian University of Athens, Athens, Greece.
} 


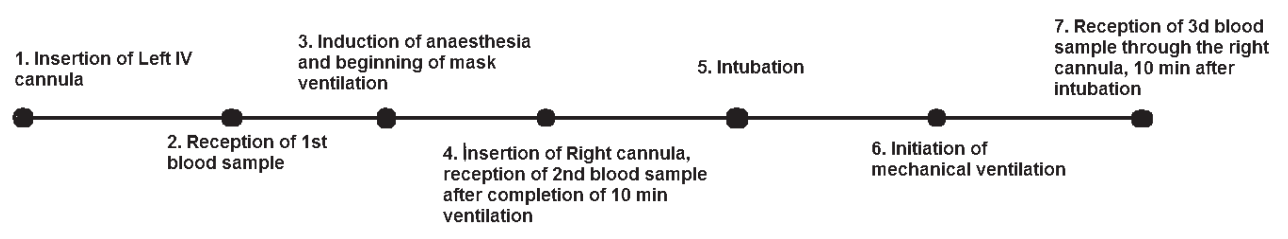

FIG. 1. Flow chart of total intervention.

addicted to opioids or alcohol, and those receiving antibiotics for any reason prior to surgery. The procedures were laparoscopic cholecystectomy $(\mathrm{N}=17)$, open cholecystectomy $(\mathrm{N}=5)$, abdominal hernia repair $(\mathrm{N}=8)$, mastectomy $(\mathrm{N}=$ $15)$, and fascial reconstruction $(\mathrm{N}=5)$. During the preoperative evaluation, an informed consent document was signed, and the airway was classified according to the Mallampati criteria [6]. The patients fasted for $8 \mathrm{~h}$ before surgery but were allowed to drink clear liquids up to $4 \mathrm{~h}$ before the time scheduled for surgery.

A eutectic mixture of lidocaine and prilocaine in the ratio 1:1 (EMLA ${ }^{\circledR}$ 5\%; AstraZeneca PLC, London, United Kingdom), was placed on two peripheral veins 40 min before the patient's admission in the operating room. Single-use anesthesia breathing circuits (Inspired Medical Technology, Istanbul, Turkey), a sterile absorbent $\mathrm{CO}_{2}$ canister, a singleuse humidifying filter (Gibeck Humid-Vent 2S Flex DC; Hudson RCI, Durham, NC), sterilized or single-use face masks (Venticare; Flexicare Medical Ltd., Mountain Ash, U.K.), endotracheal tubes of internal diameter $7.5 \mathrm{~F}$ or $8.5 \mathrm{~F}$ (Mallinckrodt, St. Louis, MO), and laryngoscope blades autoclaved at $136^{\circ} \mathrm{C}$ were used in order to prevent superinfections. Patients were ventilated by a Fabius GS anesthesia delivery system (Dräger Medical AG \& Co. KG, Lübeck, Germany) and monitored by electrocardiography, pulse oximetry, capnography, and non-invasive blood pressure measurements (Nihon Kohden, Tokyo, Japan).

Under sterile conditions, an intravenous catheter was placed in the left antecubital vein. The catheter was secured with a Tegaderm $^{\circledR}$ (3M, St. Paul, MN) film, and $10 \mathrm{~mL}$ of blood was aspirated before any other intervention using sterile gloves and proper precautions (sample 1). The sample was inoculated into $5 \mathrm{~mL}$ of aerobic and $5 \mathrm{~mL}$ of anaerobic BacT/Alert culture medium (bioMérieux, Durham, NC). At the same time, the catheter was connected to a Ringer's lactate drip by an assistant using sterile gloves and proper precautions.

Anesthesia was then induced by a 1-2 mg bolus of midazolam, lidocaine $1 \mathrm{mg} / \mathrm{kg}$, propofol 2-2.5 mg/kg, fentanyl $3-5 \mathrm{mcg} / \mathrm{kg}$, and vecuronium bromide $0.2 \mathrm{mg} / \mathrm{kg}$. All drugs were prepared in the operating theater by an assistant wearing sterile gloves. The patients were mask ventilated for 10 min while end-tidal $\mathrm{CO}_{2}$, blood pressure, and oxygen saturation were recorded constantly. A second peripheral cath- eter was then inserted into the chosen vein of the right arm, again under aseptic conditions. This catheter was used to collect samples just before intubation and after the completion of mask ventilation.

The grade of difficulty in intubation according to the Cormac and Lehane classification [7] (Grade I-IV), as well as the number of attempts required for intubation, were recorded. A first-year resident who had completed at least 50 intubations was allowed two attempts. A third attempt, if needed, was performed by the attending anesthesiologist. The last blood sample (sample 3) was collected from the right peripheral cannula $10 \mathrm{~min}$ after intubation and before any surgical intervention using sterile gloves and same precautions as previously. A flow chart of the interventions is shown in Figure 1.

The blood samples were incubated for $120 \mathrm{~h}$ in the Bactec culture system. Positive samples were recultured on blood, McConkey, and Sabouraud agar and incubated for $48 \mathrm{~h}$ at $37^{\circ} \mathrm{C}$ under aerobic conditions on chocolate agar. The samples also were incubated in $5 \% \mathrm{CO}_{2}$ for $48 \mathrm{~h}$. The samples from positive anaerobic vials were recultured on Scheedler + $5 \%$ sheep blood agar and incubated for seven days under anaerobic conditions. Bacterial identification was performed by standard methods with a mini Api system (bioMérieux). Peptostreptococcus was identified by gram stain and a susceptibility test using SPS disks.

Data were analyzed using SPSS 13.0 for Windows (SPSS, Inc., Chicago, IL). Descriptive statistics, including the mean, median, ranges, and standard deviations, were calculated for all the continuous baseline demographic and laboratory characteristics. Correlation of post-intubation bacteremia with the type of tracheal intubation, age, type of ventilation, and pre-intubation cultures was evaluated with the $\chi^{2}$ test. Differences were considered significant at $\mathrm{p}<0.05$.

\section{Results}

Fifty patients were enrolled. Their mean age was $57.6 \pm$ 15.1 years (median 57.5 years; range $20-80$ years). Only five of the 50 intubations $(10 \%)$ were considered difficult (Cormack and Lehane classification score $>2$ ).

A total of 150 blood cultures were obtained. All cultures received before and $10 \mathrm{~min}$ after the induction of anesthesia were negative $(100 \%)$. In six patients $(12 \%)$, the cultures re-

Table 1. Type of Intubation and Blood Culture Findings

\begin{tabular}{lccr}
\hline Type of intubation & $\begin{array}{c}\text { Positive blood culture } \\
(\%)\end{array}$ & $\begin{array}{c}\text { Negative blood } \\
\text { culture (\%) }\end{array}$ & Total \\
\hline Easy & $1(2.2)$ & $44(97.8)$ & 45 \\
Difficult & $5(100)$ & 0 & 5 \\
Total & $6(12.0)$ & $44(88.0)$ & 50 \\
\hline
\end{tabular}


Table 2. Pathogens Isolated from Blood Samples of Patients with Difficult Intubation

\begin{tabular}{ll}
\hline & Number of patients (\%) \\
\hline Peptostreptococcus & $1(20)$ \\
Escherichia coli & $2(40)$ \\
Staphylococcus aureus & $2(40)$ \\
Total & 5 \\
\hline
\end{tabular}

ceived $10 \mathrm{~min}$ after intubation were positive. Among the 45 patients with easy intubation, in only one case $(2.2 \%)$ was the post-intubation blood culture positive (Staphylococcus aureus) (Table 1). From patients with difficult intubations, three different organisms were isolated (Table 2). A statistically significant correlation was demonstrated between bacteremia and difficult or traumatic intubation $(\mathrm{p}=0.000$; Fisher exact test). No relation was found between post-intubation bacteremia and patient age, difficulty of intubation, or type of ventilation.

The post-intubation culture results in correlation with the type of intubation were plotted (Fig. 2). Those plots demonstrate that the majority of patients who had a positive culture after intubation had a difficult, in most cases traumatic, intubation.

\section{Discussion}

In this study, the incidence of difficult intubation, defined by the need for more than one attempt by the resident and the attending anesthesiologist, was $10 \%$, in accordance with the incidence in previous studies [1]. In prior studies, the preoperative Mallampati classification did not correlate totally with the Cormac and Lehane [7] classification because of the low sensitivity (60\%) and specificity (72\%) of the former classification. The Mallampati score, based on subjective criteria, does not predict difficult intubation accurately $[8,9]$. In our study, three of five patients with difficult intubation (Cormac and Lehane score $>2$ ) were not identified by the Mallampati score. For the residents [10], 27 cases or a workload of a mean of 57 intubations is recommended to achieve a success rare of $90 \%$ [11].

A difficult intubation may produce pressure on the oropharyngeal mucosa resulting in laceration and bleeding. In our series, blood was observed on the laryngoscopy blade in all but one case of difficult intubation, suggesting mucosal trauma. In cases where a positive blood culture was obtained, the intubation was traumatic, indicating that trauma to the mucosa might have initiated bacteremia. On the other hand, all cultures received before intubation were negative, and only one positive culture was received from a patient having a non-traumatic intubation. The issue of postoperative infections resulting from post-intubation bacteremia was not addressed in our protocol. Such investigation would have been interesting but possibly inconclusive, because antibiotics were given postoperatively at the surgeon's request.

The postoperative course of the patients was followed, and we collected the following results. A patient undergoing mastectomy, in whom $S$. aureus had been detected after intubation, presented with fever three days later, and a surgical infection was diagnosed. Unfortunately, no culture was received. Two of the patients with an easy intubation, in whom no bacteremia had been detected, likewise presented a few days later with fever. One fever was attributed to laparoscopic cholecystectomy and the other to a small area of atelectasis after abdominal hernia repair.

The problem of blood and sputum contamination of anesthesia equipment [12], in particular laryngoscope blades and handles, has been discussed extensively in the literature $[13,14]$, and yet no solution has been suggested. To avoid contamination, the supervising anesthetist took care that the tube was handled properly and was not left on any surface other than its packaging. In three cases, to avoid contamination, the endotracheal tube was changed before the second intubation attempt because of mishandling. Phillips and Monaghan reported that occult blood was present on $20 \%$ of laryngoscope blades and $40 \%$ of laryngoscope handles that were identified as ready for patient use [15]. In our study, laryngoscope blades, handles, and soda canisters were autoclaved before use in every patient.

The incidence of bacteremia after endotracheal intubation has been examined in earlier studies [16-18] and was reported to be $0-5.3 \%$. In a series of 62 patients, Goldstein et al. found an incidence of bacteremia of 3.2\% [2]. The incidence of bacteremia in other procedures such as nasotracheal intubation is higher $[18,19]$. The incidence of bacteremia in our study (12\%) cannot be attributed to contamination during the procedure, as all equipment was sterilized, and all staff members used sterile gloves in every intervention, including IV cannulation, endotracheal intubation, and blood aspiration for bacterial culture or any other purpose. No one ever used non-sterile gloves, and all cultures obtained before intubations were negative. Furthermore, the occurrence of bacteremia showed no correlation with medication infusions, as all drugs were prepared and preserved in the operating theater and their storage was according to the

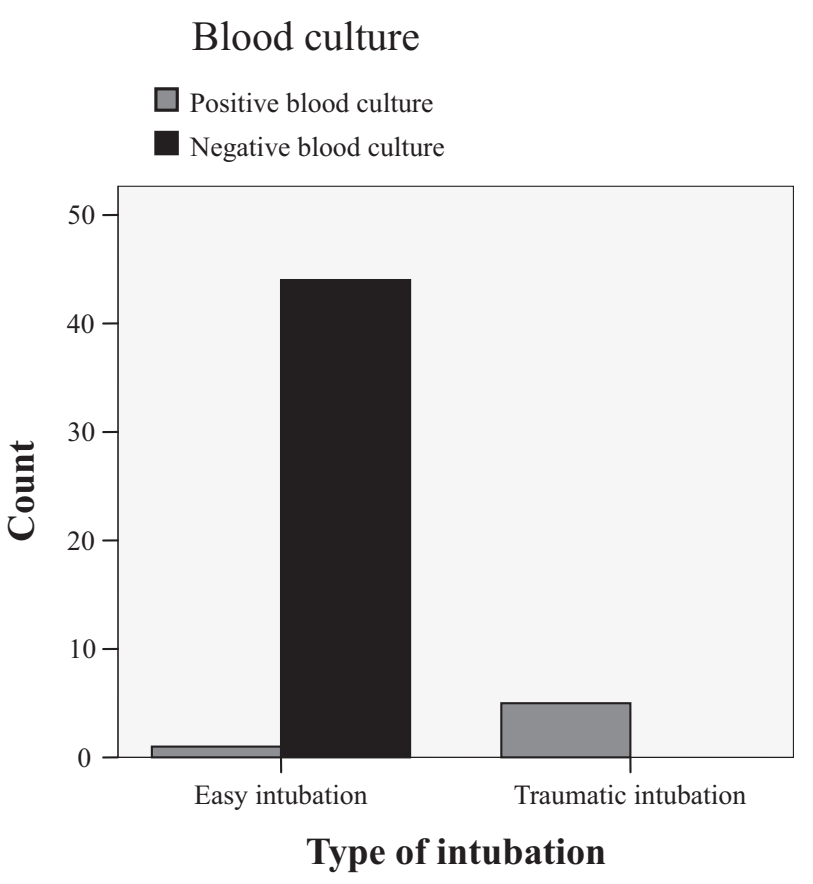

FIG. 2. Blood cultures and type of intubation. 
manufacturer's instructions. In patients with easy intubation, the incidence of bacteremia was low $(2.2 \%)$, whereas in patients with difficult intubation, it was much higher $(10 \%)$, suggesting an association between bacteremia and trauma to the oropharynx.

The time at which cultures were obtained after intubation might be important. In prior studies $[16,17]$, the samples were taken $5 \mathrm{~min}$ after intubation. In studies where the cultures were drawn $10 \mathrm{~min}$ after intubation, Gerber et al. [18] and Goldstein et al. [2] reported a higher incidence of bacteremia, but still less than that we found in difficult intubations.

The possibility of contamination of the cultures was excluded, as all positive samples were recultured. Moreover, the strains isolated do not suggest contamination. The implications of bacteremia for the postoperative course of these patients are not known, because the study was not designed to be extended into the postoperative period, and antibiotics were administered after the samples were taken whenever there was an indication.

\section{Conclusion}

Intubation resulted in bacteremia in $12 \%$ of the patients in this series. Difficult or traumatic intubation had a strong correlation with bacteremia, whereas the incidence of bacteremia in patients with easy intubation was much lower. The strains isolated were E. coli, S. aureus, and Peptosteptococcus. On the basis of these findings, difficult intubation (two or more attempts) might be a reason for antibiotic prophylaxis. Further studies are needed to strengthen this conclusion.

\section{Acknowledgments}

The authors thank Mrs. Karachaliou Eleni, microbiology technician, for her assistance. The study was fully supported financially by Evgenidion University Hospital and the Special Account of the National and Kapodistrian University of Athens.

\section{References}

1. Mrazova M, Docze A, Buckova E, et al. Prospective national survey of viridans streptococcal bacteraemia: Risk factors, antibacterial susceptibility and outcome of 120 episodes. Scand J Infect Dis 2005;37:637-641.

2. Goldstein S, Wolf GL, Kim SJ, et al. Bacteraemia during direct laryngoscopy and endotracheal intubation: A study using a multiple culture large volume technique. Anaesth Intensive Care 1997;25:239-244.

3. Ansley JF, Shapiro NL, Cunningham MJ. Rigid tracheobronchoscopy-induced bacteremia in the pediatric population. Arch Otolaryngol Head Neck Surg 1999;125:774-776.

4. Latchumana Dhas $\mathrm{K}$, Hemalatha $\mathrm{R}$, Umesan $\mathrm{CV}$, et al. Prospective evaluation of the risk of bacteraemia induced by transesophageal echocardiography. Indian Heart J 2002;54: 181-183.

5. Oncag O, Cokmez B, Aydemir S, Balcioglu T. Investigation of bacteraemia following nasotracheal intubation. Paediatr Anaesth. 2005;15:194-198.

6. Mallampati SR. Clinical sign to predict difficult tracheal intubation (hypothesis). Can Anaesth Soc J 1983;30:316.

7. Cormack RS, Lehane J. Difficult tracheal intubation in obstetrics. Anaesthesia 1984;39:1105-1111.

8. Bergler W, Maleck W, Baker-Schreyer A, et al. The Mallampati score: Prediction of difficult intubation in otolaryngologic laser surgery by Mallampati score. Anaesthesist 1997;46:437-440.

9. Butler PJ, Dhara SS. Prediction of difficult laryngoscopy: An assessment of the thyromental distance and Mallampati predictive tests. Anaesth Intensive Care 1992;20:139-142.

10. Charuluxananan S, Kyokong O, Somboonviboon W, Pothimamaka S. Learning manual skills in spinal anesthesia and orotracheal intubation: Is there any recommended number of cases for anaesthesia residency training program? J Med Assoc Thai 2001;84:S251-S255.

11. Konrad C, Schiipfer G, Markus Wietlisbach M, Gerber H. Learning manual skills in anaesthesiology: Is there a recommended number of cases for anaesthetic procedures? Anesth Analg 1998;86:635-639.

12. Hall JR. Blood contamination of anesthesia equipment and monitoring equipment. Anesth Analg 1994;78:1136-1139.

13. Skilton RWH. Risks of cross infection associated with anesthesia; cleaning procedures for laryngoscopes-A need for Association guidelines? Anaesthesia 1996;51:512-513.

14. Hall JR. Blood contamination of anesthesia equipment and monitoring equipment. Anesth Analg 1994;78:1136-1139.

15. Phillips RA, Monaghan WP. Incidence of visible and occult blood on laryngoscope blades and handles. AANAJ 1997; 65:241-246.

16. Berry FA, Blackenbaker WL, Ball CG. A comparison of bacteremia occurring with nasotracheal and orotracheal intubation Anesth Analg 1973;52:873-876.

17. Peroix JP, Malbezin S, Videcoq M, et al. Oral intubation v nasal intubation in adult cardiac surgery. $\mathrm{Br} \mathrm{J}$ Anaesth 1987;59:167-169.

18. Gerber MA, Gastanaduy AS, Buckley JJ, Kaplan EL. Risk of bacteremia after endotracheal intubation for general anesthesia. South Med J 1980;73:1478-1480.

19. Hansen $\mathrm{CP}$, Westh $\mathrm{H}$, Brok KE, et al. Bacteremia following orotracheal intubation and oesophageal balloon dilation. Thorax 1989;44:684-685.

Address reprint requests to: Dr. Evangelos A. Konstantinou 18 Kivelis str. 15238 Halandri, Athens, Greece

E-mail: ekonstan30@yahoo.com 